\title{
Cobertura do exame de Papanicolaou no Brasil e seus fatores determinantes: uma revisão sistemática da literatura
}

\author{
Coverage of the Pap smear in Brazil and its determining factors: a systematic literature review
}

\author{
Luís Felipe Leite Martins ${ }^{1}$, Luiz Claudio Santos Thuler ${ }^{2}$, Joaquim Gonçalves Valente ${ }^{3}$
}

\section{RESUM0}

Objetivo: apresentar o panorama da cobertura do exame de Papanicolaou no Brasil, com ênfase nos seus fatores determinantes. Métodos: foram pesquisadas as bases de dados "on line” LILACS (Literatura Latino-Americana e do Caribe em Ciências da Saúde), MEDLINE - 1966 a 2004 (Literatura Internacional em Ciências da Saúde), PAHO (Acervo da Biblioteca da Organização PanAmericana de Saúde) e WHOLIS (Sistema de Informação da Biblioteca da OMS). A revisão foi ampliada por meio da busca a referências bibliográficas dos estudos relevantes, solicitação de estudos publicados e não publicados a especialistas e outras fontes. Foram selecionados artigos que preenchiam os seguintes critérios: ser um estudo transversal, ter sido realizado no Brasil, conter informações sobre a cobertura do exame Papanicolaou (alguma vez na vida ou nos últimos três anos) ou sobre seus fatores determinantes. Foram excluídas as duplicidades e os artigos sem resumo. Um total de 13 artigos foram selecionados atendendo a esses critérios. Resultados: são poucos os estudos sobre a cobertura do exame de Papanicolaou no Brasil. A maioria concentrase nas grandes cidades das regiões Sul e Sudeste do país. Além da escassez, existe pouca padronização metodológica em relação à amostragem e perfil das mulheres a serem investigadas, o que torna difícil a comparação entre si. Estas diferenças metodológicas devem ter contribuído para a grande variabilidade nas coberturas encontradas. Entretanto, apesar de todos os problemas, observa-se tendência de aumento temporal no percentual de mulheres que foram submetidas a pelo menos um exame de Papanicolaou: os dois únicos estudos realizados nos anos 80 mostram coberturas de 55,0 e 68,9\% na vida, ao passo que um inquérito domiciliar realizado em 15 capitais e no Distrito Federal entre 2002 e 2003 apresenta valores que variam de 73,4 a 92,9\%; porém, dois estudos de abrangência nacional realizados em 2003 apresentaram coberturas abaixo de 70,0\% nos últimos três anos. Por outro lado, algumas variáveis foram mais freqüentemente observadas nas mulheres não submetidas ao exame de Papanicolaou: ter baixo nível socioeconômico, ter baixa escolaridade, ter baixa renda familiar e pertencer às faixas etárias mais jovens. Conclusão: os dados aqui apresentados apontam para desigualdades regionais na cobertura do exame de Papanicolaou na população feminina brasileira e para a necessidade de intervenção junto àqueles fatores a ela associados.

PALAVRAS-CHAVE: Neoplasias do colo do útero; Esfregaço vaginal; Estudos transversais; Brasil

\section{ABSTRACT}

Purpose: to present an overview of the coverage of the Pap smear in Brazil, emphasizing the determinant factors associated with failure of women to submit to the test. Methods: the literature was reviewed using the LILACS (Latin-American and Caribbean Literature in Sciences of the Health), MEDLINE - 1966 to 2004 (International Literature in Sciences of the Health), PAHO (Collection of the Library of the Pan-American Organization of Health), and WHOLIS (System of Information of the Library of OMS) databases. The review was enlarged through the search of bibliographical references of relevant studies, request for published and unpublished studies by specialists, and other sources. Articles that fulfilled the following criteria were selected: to be a cross-sectional study, carried out in Brazil, including information about periodicity of the Pap test (some time in life or in the last three years) and/or containing information about factors associated with failure of women to submit to the test. Duplicates and articles without summary were excluded. A total of 13 articles fulfilling these criteria were selected. Results: there are few studies on the coverage of Pap smear in Brazil. Most of them are concentrated in the big cities of the

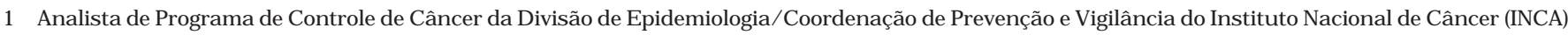
e Mestrando em Epidemiologia pelo Instituto de Medicina Social (IMS) da Universidade do Estado do Rio de Janeiro - UERJ - Rio de Janeiro (RJ), Brasil.

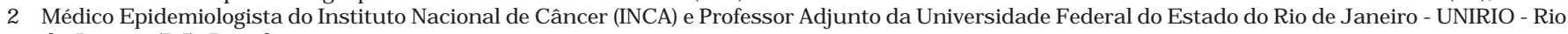
de Janeiro (RJ), Brasil.

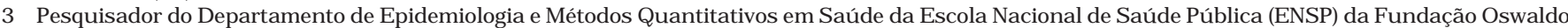
Cruz (FIOCRUZ) e Professor Adjunto do Departamento de Epidemiologia do Instituto de Medicina Social (IMS) da Universidade do Estado do Rio de Janeiro - UERJ - Rio de Janeiro (RJ), Brasil.

Correspondência: Luís Felipe Leite Martins

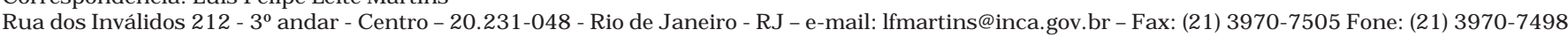

Recebido em: 18/7/2005 Aceito com modificações em: 24/8/2005 
South and Southeast regions of the country. Besides the shortage, little methodological standardization exists in relation to the sampling and profile of the investigated women, which turns difficult the comparison among them. These methodological differences must have contributed to the great variability found in the coverage. However, in spite of all of the problems, a trend of time series increase is observed in the percentage of women who had at least one Pap smear in life. The two studies accomplished in the eighties showed coverage of 55.0 and 68.9\% some time in life, while a household survey carried out in 2002 and 2003 presented values that varied from 73.4 to 92.9\%; however, two studies of national inclusion presented estimates below $70.0 \%$ in the last three years. On the other hand, some variables were associated with the women's failure to submit to the Pap smear: low socioeconomic level, low education, low family income, and to belong to the younger age groups. Conclusion: the data here presented point to regional inequalities in the coverage of the Pap smear in the Brazilian female population and to the need of intervention targeted to those factors associated with women's failure to submit to the Pap smear.

KEYWORDS: Cervix neoplasms; Vaginal smears; Cross-sectional studies; Brazil

\section{Introdução}

De forma geral, o câncer do colo do útero corresponde a cerca de $15 \%$ de todos os tipos de câncer em mulheres, sendo atualmente o segundo câncer mais comum, no sexo feminino, no mundo. Em alguns países em desenvolvimento, ocupa a primeira posição na classificação de todos os cânceres entre as mulheres, ao passo que, em países desenvolvidos, atinge o sexto lugar. As mais altas taxas de incidência de câncer do colo do útero são observadas na América Latina e países do Caribe, partes da África e no Sul e Sudeste asiático, ao passo que na América do Norte, Austrália, Norte e Oeste europeu essas são baixas ${ }^{1}$. No Brasil, as taxas de incidência ajustadas por idade variam entre 14,3 por 100.000 mulheres em Salvador e 50,7 por 100.000 mulheres no Distrito Federal ${ }^{2}$.

As informações do Sistema de Informação sobre Mortalidade mostram que, em 2002, no Brasil, o câncer de colo do útero foi responsável por $7,1 \%$ de todas as mortes por câncer em mulheres, ocupando a quarta posição ${ }^{3}$. As taxas ajustadas de mortalidade por câncer do colo do útero continuam moderadamente altas no país e, do ponto de vista temporal, vêm se mantendo estáveis: em 1979 , a taxa era de $4,97 / 100.000$, ao passo que, em 2002, era de 5,03/100.000, correspondendo a uma variação percentual relativa de $1,2 \%{ }^{4}$.

Apesar do conhecimento cada vez maior nesta área, a abordagem mais efetiva para o controle do câncer do colo do útero continua sendo o rastreamento por meio do exame preventivo de Papanicolaou. Trata-se de exame rápido, relativamente de baixo custo e efetivo para a sua detecção precoce. No entanto, sua técnica de realização é vulnerável a erros de coleta e de preparação da lâmina e a subjetividade na interpretação dos resultados. Por estas razões, os estudos têm apresentado grande variabilidade nas estimativas de sensibilidade e especificidade do exame. Uma meta-análise realizada por Fahey et al. ${ }^{5}$ apresen- tou variação de 11 a 99\% para a sensibilidade e de 14 a 97\% para a especificidade. Já a revisão sistemática realizada por Nanda et $a{ }^{6}{ }^{6}$ apresentou as seguintes variações: 30 a $87 \%$ para a sensibilidade e de $86 \%$ a $100 \%$ para a especificidade.

Uma vez que o câncer do colo do útero tem longa fase pré-clínica e o exame de Papanicolaou deve ser repetido periodicamente, espera-se que as lesões precursoras que não tenham sido identificadas num exame o sejam em ocasiões subseqüentes.

A medida da efetividade do exame de Papanicolaou em reduzir taxas de morbimortalidade por câncer do colo do útero vem de duas fontes: de estudos comparativos de tendências temporais, que têm mostrado redução nas taxas de incidência em alguns países, seguida à introdução de programas populacionais de rastreamento ${ }^{7,8}$, e de estudos epidemiológicos do tipo caso-controle, que têm indicado um risco elevado de desenvolver câncer do colo do útero entre mulheres que nunca foram submetidas ao exame de Papanicolaou, além de aumento no risco proporcional ao tempo decorrido desde o último exame ${ }^{9,10}$.

Gustafsson et al. ${ }^{7}$ trabalharam com os dados dos registros de câncer de 17 países e observaram o comportamento da taxa de incidência do câncer do colo do útero ajustada por idade, antes e depois da introdução do rastreamento. Em 11 países a redução foi marcante, variando de $27 \%$ na Noruega a $77 \%$ na Finlândia. Nas outras seis populações a redução foi inferior a $25 \%$. Por sua vez, Day ${ }^{8}$ mostrou aumento da proteção para o câncer de colo de útero quando existe rastreamento organizado, bem como aumento de proteção relativa quando se repete o exame um ano após um ou dois resultados negativos. Eluf-Neto et al. ${ }^{9}$ fizeram um estudo de caso-controle de base hospitalar para investigar o papel do papilomavírus (HPV) no desenvolvimento do câncer do colo de útero no Brasil e verificaram que a história prévia de coleta do exame de Papanicolaou foi associada ao decréscimo no risco de ter o câncer do colo do útero. Em outro estudo do tipo caso-controle, Herrero et al. ${ }^{10}$ mostraram que 
o risco relativo associado à ausência do exame foi aproximadamente de três. Mulheres submetidas ao exame há muito tempo também tiveram um risco elevado, porém menor que as demais.

No Brasil, desde 1988, o Ministério da Saúde (MS) tem seguido a recomendação da Organização Mundial de Saúde que propõe um exame de Papanicolaou a cada três anos em mulheres entre 25 e 60 anos de idade, após dois controles anuais consecutivos com resultado normal ${ }^{11}$. Mais recentemente, em 1996, o MS, por meio do Instituto Nacional de Câncer (INCA), implementou o Programa "Viva Mulher", envolvendo cinco capitais brasileiras e um estado, tendo como população-alvo, mulheres pertencentes à faixa etária de 35 a 49 anos $^{12}$. Em 1998, as ações do "Viva Mulher" foram estendidas a todos os municípios brasileiros por meio de uma campanha nacional. Desde então, tem-se observado crescente ampliação da oferta de exames citopatológicos no país: antes de 1998 o número de exames realizados não ultrapassava 7 milhões por ano. Em 1998, ano em que houve a campanha, esse número passou para 10,3 milhões. De 1999 a 2001 foram processados na rotina, em média, 7,8 milhões por ano e em 2002, ocorreu uma nova intensificação da oferta de exames visando aumentar a sua cobertura, resultando em 12,2 milhões de exames; no período de 2003 a 2004 foram realizados na rotina, em média, 10,4 milhões de exames por ano ${ }^{13}$.

No entanto, apesar dos esforços crescentes para melhorar a eficiência dos programas de prevenção, ou seja, aumentar o número de mulheres examinadas com qualidade e com o menor custo possivel, a manutenção de altas taxas de incidência e de mortalidade no Brasil revela que as medidas que vêm sendo adotadas não conduziram aos resultados esperados.

No Brasil, há poucas estimativas sobre a cobertura das ações de detecção precoce do câncer do colo do útero na população feminina. Na década de 80 alguns estudos consideraram o número de exames citopatológicos feitos anualmente. Aquino ${ }^{14}$ realizou uma pesquisa entre 1980 e 1983, e constatou que a cobertura do exame de Papanicolaou era de $1,2 \%$ nas mulheres com mais de 15 anos. Contudo, a pesquisa só utilizou dados das secretarias estaduais de saúde e da Fundação FESP, faltando assim informações detalhadas sobre as atividades do INAMPS, que era o sistema de saúde que vigorava na época. Baseado em levantamento de dados recebidos dos laboratórios de citopatologia, em 1987, foi estimado pelo Registro Nacional de Patologia Tumoral que $7,7 \%$ das mulheres brasileiras com mais de 20 anos já tinham tido acesso ao teste de Papanicolaou ${ }^{15}$. Também por meio de dados de laboratórios, Zeferino ${ }^{16}$ estimou para o Estado de São Paulo cobertura de $19,6 \%$ para o ano de 1986 e 25,0 e $30,0 \%$ para os dois anos posteriores.
A revisão sistemática da literatura aqui apresentada tem como objetivo descrever o panorama da cobertura do exame de Papanicolaou no Brasil, com ênfase na identificação dos seus determinantes.

\section{Métodos}

A revisão sistemática da literatura foi realizada no segundo semestre de 2004 a partir das bases de dados on-line LILACS (Literatura Latino-Americana e do Caribe em Ciências da Saúde), MEDLINE - 1966 a 2004 (Literatura Internacional em Ciências da Saúde), PAHO (Acervo da Biblioteca da Organização Pan-Americana da Saúde) e WHOLIS (Sistema de Informação da Biblioteca da OMS). Foram utilizadas as seguintes palavras-chave (em português e inglês): Papanicolaou, citopatologia, citopatológico, colpocitologia, exame preventivo ou esfregaço vaginal e cobertura, fatores, razões ou motivos e Brasil. Estas bases foram acessadas por meio da Biblioteca Virtual em Saúde Pública (BVS) / Bireme/OPAS/OMS (http://bases.bvs.br). A revisão foi ampliada por meio da busca a referências bibliográficas dos estudos relevantes, solicitação de estudos publicados ou não a especialistas e busca em outras fontes. O total de artigos resultantes da busca, conforme as palavras-chave utilizadas, para cada base de dados pode ser observado na Quadro 1 .

Quadro 1 - Referências bibliográficas identificadas conforme as palavras-chave e bases de dados pesquisadas, 1966 a 2004.

\begin{tabular}{|c|c|c|}
\hline Palavras-chave & $\begin{array}{c}\text { Bases } \\
\text { de dados }\end{array}$ & $\begin{array}{l}\text { Referências } \\
\text { identificadas }\end{array}$ \\
\hline $\begin{array}{l}\text { Papanicolaou OR Citopatologia OR } \\
\text { Citopatológico OR Colpocitologia OR } \\
\text { Exame Preventivo OR Esfregaço Vaginal } \\
\text { AND Cobertura OR Fatores OR Razões } \\
\text { OR Motivos }\end{array}$ & LILACS & 105 \\
\hline $\begin{array}{l}\text { Papanicolaou OR Cytopathology OR } \\
\text { Cytopathological OR Colpocytology OR } \\
\text { Preventive Exam OR Vaginal Smears } \\
\text { AND Coverage OR Factors or Reasons } \\
\text { OR Motives }\end{array}$ & LILACS & 68 \\
\hline $\begin{array}{l}\text { Papanicolaou OR Citopatologia OR } \\
\text { Citopatológico OR Colpocitologia OR } \\
\text { Exame Preventivo OR Esfregaço Vaginal } \\
\text { AND Cobertura OR Fatores OR Razões } \\
\text { OR Motivos AND Brasil }\end{array}$ & $\begin{array}{c}\text { MEDLINE } \\
\text { PAHO } \\
\text { WHOLIS }\end{array}$ & 20 \\
\hline $\begin{array}{l}\text { Papanicolaou OR Cytopathology OR } \\
\text { Cytopathological OR Colpocytology OR } \\
\text { Preventive Exam OR Vaginal Smears } \\
\text { AND Coverage OR Factors OR Reasons } \\
\text { OR Motives AND Brazil. }\end{array}$ & $\begin{array}{l}\text { MEDLINE } \\
\text { PAHO } \\
\text { WHOLIS }\end{array}$ & 27 \\
\hline $\begin{array}{l}\text { Especialistas e referências bibliográficas } \\
\text { de textos e artigos }\end{array}$ & - & 7 \\
\hline
\end{tabular}


Foram selecionados artigos que preenchiam os seguintes critérios: ser um estudo transversal, ter sido realizado no Brasil, conter informações sobre a cobertura do exame de Papanicolaou (alguma vez na vida ou nos últimos três anos) ou conter informações sobre seus determinantes. Foram excluídas as duplicidades e os artigos sem resumo. Um total de 13 artigos foram selecionados atendendo a esses critérios.

\section{Resultados}

\section{Cobertura do exame de Papanicolaou no Brasil}

Nas Tabelas 1 e 2 estão listadas as publicações sobre a cobertura do exame de Papanicolaou no Brasil. Cabe ressaltar que há diferenças metodológicas entre os estudos em relação aos seguintes aspectos: representatividade da amostra, faixa etária das mulheres entrevistadas e periodicidade do exame (alguma vez na vida ou nos últimos três anos).

Dos 13 estudos, nove utilizaram delineamento transversal de base populacional, baseando-se em amostra aleatória representativa da população, e quatro utilizaram delineamento transversal a partir de amostra não aleatória. A seguir apresentaremos separadamente os resultados obtidos por estes dois grupos de estudos.

\section{Estudos transversais baseados em amostras probabilisticas (Tabela 1)}

Tabela 1 - Estudos transversais baseados em amostras probabilísticas sobre a cobertura do exame de Papanicolaou, Brasil, 1987-2003.

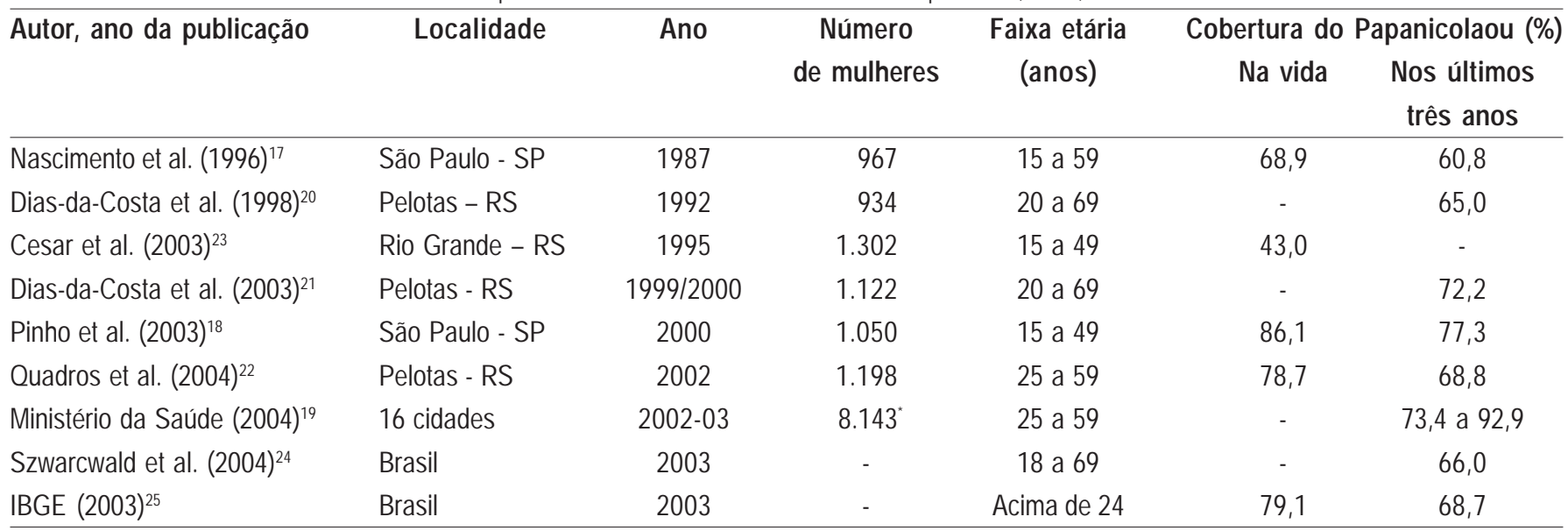

* - Somatório das amostras das 16 cidades

No município de São Paulo foram realizados três estudos transversais. O primeiro deles, referente ao ano de 1987, incluiu 967 mulheres de 15 a 59 anos. Destas, $68,9 \%$ haviam feito o exame alguma vez na vida, ao passo que $60,8 \%$ o haviam feito nos três anos anteriores à pesquisa ${ }^{17}$. O segundo estudo, realizado em 2000, incluiu 1.172 mulheres de 15 a 49 anos. Das mulheres que já tinham iniciado a vida sexual $(n=1.050), 86,1 \%$ tinham sido submetidas ao exame alguma vez na vida e $77,3 \%$ nos três anos anteriores à pesqui$\mathrm{sa}^{18}$. O último estudo foi um inquérito domiciliar realizado em 2003. De 447 mulheres de 25 a 59 anos, $81,4 \%$ referiram ter pelo menos um exame de Papanicolaou nos três anos que antecederam à entrevista. Este inquérito foi também realizado em outras 15 cidades brasileiras (Belém, Manaus, Aracaju, Fortaleza, João Pessoa, Natal, Recife, Campo Grande, Distrito Federal, Belo Horizonte, Rio de Janeiro, Vitória, Curitiba, Florianópolis e Porto Alegre). O percentual de mulheres submeti- das ao exame nos três anos que antecederam a coleta dos dados variou de 73,4\% em João Pessoa a 92,9\% em Vitória ${ }^{19}$.

Além dessas localidades, a área urbana do município de Pelotas (RS) foi alvo de três estudos de base populacional. O primeiro deles, ocorrido em 1992, baseou-se em amostra composta de 934 mulheres de 20 a 69 anos, das quais $65,0 \%$ haviam feito o exame de Papanicolaou nos três anos que antecederam a coleta dos dados ${ }^{20}$. O segundo estudo, realizado entre 1999 e 2000, referia-se a uma amostra constituída de 1.122 mulheres entre 20 e 69 anos, sendo que $72,2 \%$ informaram ter sido submetidas ao exame dentro do prazo preconizado, ou seja, há menos de três anos, enquanto $11,2 \%$ nunca o haviam feito; as demais $16,6 \%$ informavam ter realizado o último exame há mais de três $\operatorname{anos}^{21}$. O último estudo ocorreu em 2002, baseando-se em amostra composta de $1.198 \mathrm{mu}-$ lheres entre 25 e 59 anos, das quais $78,7 \%$ haviam feito o exame de Papanicolaou alguma vez na 
vida e $68,8 \%$ nos três anos anteriores à pesqui$\mathrm{sa}^{22}$.

Outro estudo, realizado no município do Rio Grande (RS), incluiu 1.302 mulheres de 15 a 49 anos, e mostrou que 57,0\% delas nunca haviam se submetido ao exame de Papanicolaou ${ }^{23}$.

Os estudos apresentados anteriormente referem-se à cobertura do exame em municípios isolados. Em 2003 foram realizados dois inquéritos de abrangência nacional. Um primeiro estudo foi a Pesquisa Mundial de Saúde 2003 realizada pela OMS em 71 países. No Brasil foram entrevistados cinco mil brasileiros com idade acima de 18 anos, residentes em 188 municípios. Esta pesquisa mostrou que a cobertura do exame de Papanicolaou (nos três anos anteriores), entre mulheres de 18 a 69 anos, foi de $66,0 \%{ }^{24}$. O segundo estudo foi realizado pelo IBGE (Instituto Brasileiro de Geografia e Estatística) por meio da PNAD (Pesquisa Nacional por Amostra de Domicílios) Saúde 2003, e mostrou que a cobertura do exame de Papanicolaou foi de $68,7 \%$ nas mulheres com mais de 24 $\operatorname{anos}^{25}$.

Estudos transversais baseados em amostras não probabilisticas (Tabela 2)

Tabela 2 - Estudos transversais baseados em amostras não probabilísticas sobre a cobertura do exame de Papanicolaou, Brasil, $1988-2002$.

\begin{tabular}{|c|c|c|c|c|c|c|}
\hline Autor, ano da publicação & Localidade & Ano & $\begin{array}{c}\text { Número } \\
\text { de mulheres }\end{array}$ & $\begin{array}{l}\text { Faixa etária } \\
\text { (anos) }\end{array}$ & $\begin{array}{c}\text { Cobertura do } \\
\text { Na vida }\end{array}$ & $\begin{array}{c}\text { Papanicolaou (\%) } \\
\text { Nos últimos } \\
\text { três anos }\end{array}$ \\
\hline Pinotti et al. $(1990)^{26}$ & São Paulo & 1988 & 3.703 & 15 a 49 & 53,1 & - \\
\hline \multirow[t]{5}{*}{ Lopes et al. $(1995)^{27}$} & Região Norte & 1994 & 1.478 & & 60,0 & - \\
\hline & Região Nordeste & & & $\geq 16$ & 58,0 & - \\
\hline & Região Sudeste & & & & 69,0 & - \\
\hline & Região Sul & & & & 62,0 & - \\
\hline & Região Centro Oeste & & & & 60,0 & - \\
\hline IBOPE $(2002)^{28}$ & São Paulo & 2002 & 2.300 & 20 a 69 & 90,0 & - \\
\hline Governo do Estado do Paraná (2002)29 & Paraná & 2002 & 2.007 & $\geq 16$ & - & $75,0^{*}$ \\
\hline
\end{tabular}

${ }^{\star}$ Nos últimos 5 anos.

Em 1988, a Secretaria Estadual de Saúde de São Paulo conduziu uma pesquisa baseada em 2.021 mulheres de 15 a 49 anos. Foram entrevistadas mulheres residentes na região metropolitana e no interior do Estado. Observou-se que $53,1 \%$ tinham sido submetidas ao exame de $\mathrm{Pa}-$ panicolaou alguma vez na vida, enquanto $88,8 \%$ tinham-no realizado nos três anos anteriores à pesquisa $^{26}$.

Por solicitação do INCA, em 1994, o Instituto Brasileiro de Opinião e Pesquisa (IBOPE) realizou um inquérito com uma amostra referente às grandes regiões do Brasil, na qual se constatou que $36,0 \%$ das mulheres entrevistadas nunca haviam feito o exame de Papanicolaou. Os percentuais variavam entre $31 \%$ na região $\mathrm{Su}-$ deste e $42 \%$ na região Nordeste ${ }^{27}$.

Além desse último estudo, o IBOPE realizou, em 2002, uma outra pesquisa envolvendo a população feminina de 20 a 69 anos residente no Estado de São Paulo. A análise dos resultados baseados em amostra constituída de 2.300 mulheres mostrou que $89,0 \%$ realizaram pelo menos um exame de Papanicolaou na vida ${ }^{28}$. Neste mesmo ano, foi conduzida uma pesquisa no estado do Paraná, que revelou que $75,0 \%$ das mulheres en- trevistadas haviam sido submetidas ao exame nos cinco anos anteriores à entrevista ${ }^{29}$.

Fatores associados à não submissão ao exame de Papanicolaou em mulheres brasileiras

Quadro 2 - Principais fatores associados à não submissão ao exame de Papanicolaou em mulheres brasileiras.

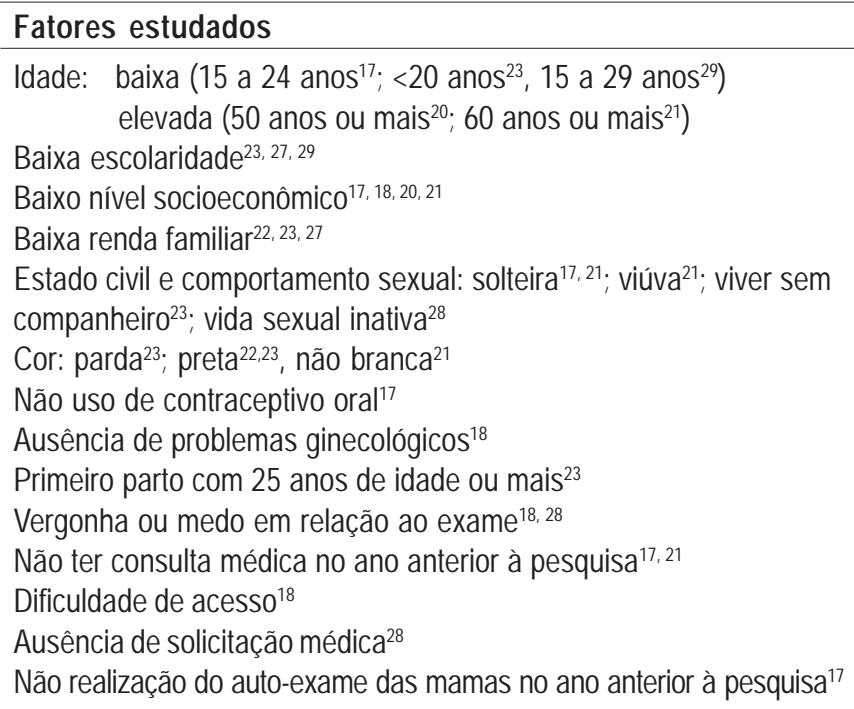


No Quadro 2 encontra-se uma síntese dos estudos que analisaram os fatores para a não submissão ao exame de Papanicolaou no território nacional. No estudo realizado em 1987 por Nascimento et al. ${ }^{17}$ os seguintes fatores foram apontados: ter entre 15 e 24 anos de idade, ser solteira, ter baixo nível socioeconômico, não ter consultado o médico no ano anterior à pesquisa, não ter usado contraceptivos orais e não ter realizado o auto-exame das mamas no último ano.

Já no estudo realizado por Pinho et al. ${ }^{18}$ observou-se que os principais motivos foram: não ter problemas ginecológicos, ter vergonha ou medo de realizar o exame e referir dificuldade de acesso à unidade de saúde. Este estudo mostrou ainda que a proporção de mulheres que referiu ter sido submetida ao exame de forma espontânea (sem recomendação médica e sem problemas ginecológicos) foi menor entre aquelas com baixo nível socioeconômico.

Por sua vez, Dias-da-Costa et al. ${ }^{20,21}$ concluíram que em mulheres pertencentes às classes sociais mais baixas, mais velhas, não brancas, viúvas ou solteiras, e que não consultavam regularmente o médico foi menor a freqüência do exame de Papanicolaou.

O estudo realizado por Quadros et al. ${ }^{22}$ mostrou que as mulheres de cor preta e de baixa renda realizaram proporcionalmente menos o exame preventivo.

Cesar et al. ${ }^{23}$ encontraram que as maiores razões de prevalência para não terem sido submetidas ao exame ocorreram entre as mulheres de cor parda ou preta, com idade menor do que 20 anos, com renda familiar e escolaridade baixas, vivendo sem companheiros e cujo primeiro parto foi com 25 anos de idade ou mais. Os estudos com amostras não probabilísticas concluíram que quan-

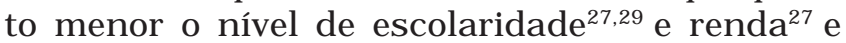
mais baixa a faixa de idade (15 a 29 anos) ${ }^{29}$, menor a cobertura do exame. A esses fatores, somamse a vergonha, ausência de solicitação médica e vida sexual inativa ${ }^{28}$.

\section{Discussão}

A partir desta revisão sistemática, observase que são poucos os estudos transversais, principalmente os baseados em amostras probabilísticas, sobre a cobertura do exame de Papanicolaou no Brasil. A maioria dos estudos encontrados concentra-se nas regiões Sul e Sudeste do país e corresponde a grandes cidades. Além dessa escassez, existe pouca padronização metodológica em relação à amostragem e perfil das mulheres a serem investigadas, o que torna difícil a comparação entre si. É importante ressaltar que estudos realizados com amostras não probabilísticas podem apresentar tendências em seus resultados, além da impossibilidade do cálculo dos erros de amostragem. Estas diferenças metodológicas podem ter contribuído para a grande variabilidade observada nas coberturas. Entretanto, apesar de todos esses problemas, há tendência de aumento temporal no percentual de mulheres submetidas a pelo menos um exame de Papanicolaou: os dois estudos realizados nos anos 80 mostram coberturas de $53,1^{26}$ e $68,9 \%{ }^{17}$ na vida, ao passo que o inquérito domiciliar realizado entre 2002 e 2003 apresenta valores que variam de 73,4 a $92,9 \%{ }^{19}$; porém, os dois estudos de base populacional com abrangência nacional ocorridos em 2003 apresentaram coberturas de menos de $70,0 \%{ }^{24,25}$ nos últimos três anos.

Para a $\mathrm{OMS}^{30}$, uma cobertura de $80 \%$ do exame entre mulheres de 35 a 59 anos seria suficiente para causar impacto nos indicadores de morbimortalidade, que pode ser observado após quatro anos de implementação das ações de detecção precoce $^{31}$. No Brasil, as taxas de mortalidade têm se mantido relativamente estáveis nos últimos anos. Entretanto, um estudo realizado em 96 centros nacionais de tratamento de câncer mostrou que o percentual de mulheres com doença avançada (estádios III e IV) no momento do diagnóstico vem diminuindo, passando de 51,7\% em 1995 para 42,5\% em 2002: uma redução de $18 \%{ }^{32}$.

Por outro lado, os fatores que levam as mulheres a não se submeter ao exame de Papanicolaou têm se repetido nos diferentes estudos: mulheres com baixo nivel socioeconômico, com baixa escolaridade, com baixa renda familiar e pertencentes às faixas etárias mais jovens. Observa-se que a maioria desses estudos trabalhou com um modelo teórico de cunho individual, enfatizando características sociodemográficas e comportamentais, sem levar em consideração outras dimensões importantes associadas à dificuldade de acesso ao exame de Papanicolaou sob um aspecto organizacional ou programático ${ }^{33}$.

Finalmente, os dados aqui apresentados apontam para desigualdades regionais na cobertura do exame de Papanicolaou na população feminina brasileira e para necessidade de intervenção junto àqueles fatores a ela associados, priorizando-se atividades de educação para o diagnóstico precoce em pessoas sintomáticas e rastreamento em mulheres assintomáticas, além da garantia de acesso aos métodos de diagnóstico e tratamento adequados. 


\section{Referências}

1. International Agency for Research on Cancer-IARC [homepage on the Internet]. IARC confirms efficacy of cervix cancer screening for women 25-65 in reducing mortality. Press release $\mathrm{n}^{\circ} 151$. Lyon; 2004 [cited 2004 May 3]. Available from: http:// www.iarc.fr/ENG/Press_Releases/Summary.pdf

2. Ministério da Saúde. Secretaria de Assistência à Saúde. Instituto Nacional de Câncer. Câncer no Brasil: dados dos registros de base populacional. Rio de Janeiro: INCA; 2003. v. 3.

3. Ministério da Saúde [sitio na Internet]. Sistema de informação sobre mortalidade. Brasília; 2005 [citado 2005 Jul 3]. Disponivel em: http:/ /www.datasus.gov.br.

4. Ministério da Saúde. Secretaria Nacional de Assistência à Saúde. Instituto Nacional de Câncer. Coordenação de Prevenção e Vigilância. Atlas de mortalidade por câncer no Brasil 1979-1999. Rio de Janeiro: INCA; 2002.

5. Fahey MT, Irwig L, Macaskill P. Meta-analysis of Pap test accuracy. Am J Epidemiol. 1995; 141(7):6809.

6. Nanda K, McCrory DC, Myers ER, Bastian LA, Hasselblad V, Hickey JD, et al. Accuracy of the Papanicolaou test in screening for and follow-up of cervical cytologic abnormalities: a systematic review. Ann Intern Med. 2000;132(10):810-9.

7. Gustafsson L, Ponten J, Zack M, Adami HO. International incidence rates of invasive cervical cancer after introduction of cytological screening. Cancer Causes Control. 1997;8(5):755-63.

8. Day NE. The epidemiological basis for evaluating different screening policies. In: Hakama M, Miller $\mathrm{AB}$, Day NE, editors. Screening for cancer of the uterine cervix. Lyon: IARC; 1986. (IARC Scientific Publication, 76).

9. Eluf-Neto J, Booth M, Muñoz N, Bosch FX, Meijer CJ, Walboomers JM. Human papillomavirus and invasive cervical cancer in Brazil. Br $\mathrm{J}$ Cancer. 1994;69(1):114-9.

10.Herrero R, Brinton LA, Reeves WC, Brenes MM, de Briton RC, Gaitan E, et al. Screening for cervical cancer in Latin America: a case-control study. Int $J$ Epidemiol. 1992;21(6):1050-6.

11. Ministério da Saúde. Secretaria Nacional de Assistência à Saúde. Instituto Nacional de Câncer. Consenso de periodicidade e faixa etária no exame de prevenção do câncer cérvico-uterino, 1988. Rev Bras Cancerol. 1989;35(1/2):77.

12. Thuler LCS. Lesões precursoras do câncer do colo uterino - aspectos epidemiológicos. In: Figueiredo EMA, editor. Ginecologia oncológica. Rio de Janeiro: Revinter; 2004. p. 3-6.
13.Ministério da Saúde. DATASUS [sitio na Internet]. Informações de saúde. Brasília; 2005 [citado 2005 Abr 14]. Disponível em: http://www.datasus.gov.br.

14.Aquino E. Avaliação da situação atual das ações de controle. Rev Bras Cancerol. 1985;31(3):185-8.

15.Abreu E. Reorientação nas ações de prevenção e controle do câncer cérvico-uterino. Rev Bras Cancerol. 1989;35(1/2):55-8.

16.Zeferino LC. Cobertura de população. Rev Bras Cancerol. 1989;35(1/2):61-2.

17. Nascimento CM, Eluf-Neto J, Rego RA. Pap test coverage in São Paulo municipality and characteristics of the women tested. Bull Pan Am Health Organ. 1996;30(4):302-12.

18.Pinho AA, França-Júnior I, Schraiber LB, D`Oliveira AFPL. Cobertura e motivos para a realização ou não do teste de Papanicolaou no município de São Paulo. Cad Saúde Pública. 2003; 19 Supl. 2:303-13.

19. Ministério da Saúde. Secretaria de Vigilância em Saúde. Secretaria de Atenção à Saúde. Instituto Nacional de Câncer. Coordenação de Prevenção e Vigilância. Inquérito Domiciliar sobre comportamentos de risco e morbidade referida de doenças e agravos não transmissíveis: Brasil, 15 capitais e Distrito Federal 2002-2003. Rio de Janeiro: INCA; 2004.

20.Dias-da-Costa JS, D' Elia PB, Manzolli P, Moreira MR. Cobertura do exame citopatológico na cidade de Pelotas, Brasil. Rev Panam Salud Publica. 1998;3(5):308-13.

21.Dias-da-Costa JS, Olinto MTA, Gigante DP, Menezes AMB, Macedo S, Borba AT, et al. Cobertura do exame citopatológico na cidade de Pelotas, Rio Grande do Sul, Brasil. Cad Saúde Pública. 2003;19(1):191-7.

22. Quadros CAT, Victora CG, Dias-da-Costa JS. Coverage and focus of a cervical cancer prevention program in southern Brazil. Rev Panam Salud Publica. 2004;16(4):223-32.

23.Cesar JA, Horta BL, Gomes G, Houlthausen RS, Willrich RM, Kaercher A, et al. Fatores associados à não realização de exame citopatológico de colo uterino no extremo Sul do Brasil. Cad Saúde Pública. 2003;19(5):1365-72.

24.Szwarcwald CL, Viacava F, Vasconcellos MTL, Leal MC, Azevedo LO, Queiroz RSB, et al. Pesquisa Mundial de Saúde 2003: o Brasil em números. RADIS. 2004;(23):14-33.

25.Instituto Brasileiro de Geografia e Estatística (IBGE) [sitio na Internet]. Pesquisa Nacional por Amostra de Domicílios. Acesso e utilização de serviços de saúde. 2003 [citado 2005 Mai 30]. Disponível em: http://www.ibge.gov.br/home/estatistica/ populacao/trabalhoerendimento/pnad2003/saude/ saúde 2003.pdf. 
26.Pinotti JA, Faúndes A, Hardy EE, Simões IR, Osis MJD, Souza TR, et al. Avaliação da assistência ginecológica no estado de São Paulo. Rev Ginecol Obstet. 1990;1(1):7-21.

27.Lopes ER, Rebelo MS, Abreu E, Costa Silva VL, Eisenberg ALA, Lavor MF. Comportamento da população feminina brasileira em relação ao câncer cérvico-uterino. J Bras Ginecol. 1995;105(11/ 12):505-15.

28.Instituto Brasileiro de Opinião Pública e EstatísticaIBOPE. Pesquisa de opinião pública com a população feminina em relação aos exames preventivos para câncer ginecológico. Sumário analítico. São Paulo; 2002.

29. Governo do Estado do Paraná. Secretaria de Estado de Saúde. Instituto de Saúde. Câncer cérvico uterino. Sumário analítico. Curitiba; 2002.
30.World Health Organization-WHO. Manual on the prevention and control of common cancers. Geneve: WHO; 1998. p. 8-31.

31. Thuler LC. Considerações sobre a prevenção do câncer de mama feminino. Rev Bras Cancerol. 2003;49(4):227-38.

32. Thuler LC, Mendonça GA. Estadiamento inicial dos casos de câncer de mama e colo do útero em mulheres brasileiras. Rev Bras Ginecol Obstet. No prelo 2005.

33.Pinho AA, França-Júnior I. Prevenção do câncer de colo do útero: um modelo teórico para analisar o acesso e a utilização do teste de Papanicolaou. Rev Bras Saúde Matern Infant. 2003;3(1):95-112. 